\title{
Electromagnetic Reciprocal Cloak with Only Axial Material Parameter Spatially Variant
}

\author{
Jing Jing Yang, Ming Huang, Fu Chun Mao, Guang Hui Cai, and Yao Zhong Lan \\ School of Information Science and Engineering, Yunnan University, Kunming 650091, China \\ Correspondence should be addressed to Ming Huang, huangming@ynu.edu.cn
}

Received 19 August 2011; Revised 10 October 2011; Accepted 10 October 2011

Academic Editor: Tat Yeo

Copyright () 2012 Jing Jing Yang et al. This is an open access article distributed under the Creative Commons Attribution License, which permits unrestricted use, distribution, and reproduction in any medium, provided the original work is properly cited.

Reciprocal cloak is an intriguing metamaterial device, in which a hidden antenna or a sensor can receive electromagnetic irradiation from the outside but its presence will not be detected. Based on transformation optics, a cylindrical electromagnetic reciprocal cloak with only axial parameter varying with radius is designed and validated by full wave simulation. When two dispersive reciprocal cloaks are put together, they do not interfere with each other. Our work demonstrates the electromagnetic compatibility (EMC) ability of the reciprocal cloak which is very important in multi antenna and sensor design.

\section{Introduction}

In the past decade, a fascinating term "metamaterials" has attracted a great deal of attentions since it provides many possibilities to explore unknown physical phenomena and fabricate novel devices [1-6] such as perfect lens, sensors, and antennae. Recently, theory of transformation optics generalized by Pendry et al. and Leonhardt $[7,8]$ built up a bridge between the function of metamaterials and material parameter distribution and provided a complete prescription for controlling and guiding electromagnetic wave as well [9-17]. One of the most popular and intriguing topic in this field is perhaps the "invisible cloak" [9-12], which makes the incident wave detour around the cloaked region without disturbance, thus an arbitrary object might be hidden inside; but the object has to be "blind," since no outside electromagnetic waves can reach within the cloaked space [18].

To overcome this limitation, some novel cloaking schemes such as open cloak and external cloak were developed. The open cloak [19-21] strategy provides an approach to open a window on the surface of a cloak, so that exchanging information and matter with the outside can be achieved. The external cloak $[22,23]$ is capable of hiding arbitrary objects at a distance outside the cloaking shell and thus share information with the surrounding. It is based on the idea of scattering cancelation, that is, the scattered field of an object is cancelled by its antiobject located nearby. Besides, scattering of a target object can also be cancelled or minimized by coating with a layer of well-designed materials. This has been firstly considered by Chew and Kerker [24]. Latterly, Alù and Engheta [25] showed that it is possible to reduce the scattering cross-section of spherical and cylindrical objects using lossless plasmonic or metamaterial covers. In the long wavelength limit, Gao et al. [26, 27] developed isotropic permittivity and permeability tensors of small coated sphere and cylinder. They demonstrated that total scattering width can be dramatically reduced by suitable adjustment of the dielectric anisotropy of the shell. In our previous work, we proposed a cloaking scheme named as reciprocal cloak [28] based on the theory of transformation optics. We show that arbitrary object can be cloaked when coated with a complementary media. Since electromagnetic waves penetrate into the cloaked space, the object hidden inside can exchange information with the outside, which may lead to many potential applications in electromagnetic engineering. However, practical fabrication of the reciprocal cloak is not so far achievable for the material parameters which are highly anisotropic and even singular. In this paper, we establish a flexible transformation procedure to design a cylindrical reciprocal cloak with only axial material parameter varying with radius, which makes it possible to be realized by layered metamaterials $[29,30]$. EMC ability of the cloak is validated by full wave simulation. The impact 


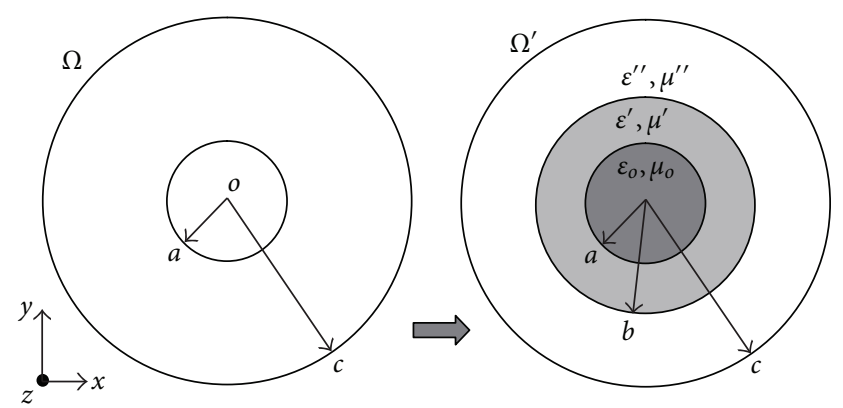

Figure 1: Schematic diagrams for describing the coordinate transformation of the reciprocal cloak.

of metamaterial loss and material parameter deviation on cloaking effect is also analyzed in detail.

\section{Method and Simulation Model}

Figure 1 shows the schematic diagram for the construction of the cylindrical reciprocal cloak. A point $O$ in the virtual space $(\Omega)$ is transformed into a circle with a radius of $b$ in the physical space $\left(\Omega^{\prime}\right)$, while keeping the inner and outer boundary unchanged. So the whole transformation space is divided into three regions, that is, the cloaked object $\left(r^{\prime}<a\right)$, the complementary media $\left(a<r^{\prime}<b\right)$, and the recovering layer $\left(b<r^{\prime \prime}<c\right)$. The working principle of the cloak can be described in two steps. First, the object is optically cancelled by the complementary media. Then, correct optical path in the canceled space is restored by the recovering layer. In the complementary media layer, we suppose the transformation function between the physical space and the virtual space is in the form of $r=f\left(r^{\prime}\right)$, where $f\left(r^{\prime}\right)$ is a continuous function of $r^{\prime}$, and it satisfies $f(b)=\delta, f(a)=a$. Here, $\delta$ is a constant that approximates zero. To avoid singularity, we set it to be 0.002 in this paper. According to the optical transformation theory and the form invariance of Maxwell's equations, relative permittivity $\varepsilon^{\prime}$ and permeability $\mu^{\prime}$ of the complementary media can be written as

$$
\begin{gathered}
\frac{\varepsilon_{r}^{\prime}}{\varepsilon_{o r}}=\frac{\mu_{r}^{\prime}}{\mu_{o r}}=\frac{f\left(r^{\prime}\right)}{r^{\prime} d f\left(r^{\prime}\right) / d r^{\prime}}, \\
\frac{\varepsilon_{\varphi}^{\prime}}{\varepsilon_{o \varphi}}=\frac{\mu_{\theta}^{\prime}}{\mu_{o \varphi}}=\frac{r^{\prime} d f\left(r^{\prime}\right) / d r^{\prime}}{f\left(r^{\prime}\right)}, \\
\frac{\varepsilon_{z}^{\prime}}{\varepsilon_{o z}}=\frac{\mu_{z}^{\prime}}{\mu_{o z}}=\frac{f\left(r^{\prime}\right) d f\left(r^{\prime}\right) / d r^{\prime}}{r^{\prime}},
\end{gathered}
$$

where $\varepsilon_{o}$ and $\mu_{o}$ are the relative permittivity and permeability of the cloaked object. We can notice that if we set (1b) as a constant, (1a) will be fixed. Thus, we suppose that

$$
\frac{\varepsilon_{\varphi}^{\prime}}{\varepsilon_{o \varphi}}=\frac{\mu_{\varphi}^{\prime}}{\mu_{O \varphi}}=\frac{r^{\prime} d f\left(r^{\prime}\right) / d r^{\prime}}{f\left(r^{\prime}\right)}=m_{0}^{\prime} .
$$

A solution of this differential equation is given by

$$
r=f\left(r^{\prime}\right)=m_{1}^{\prime} r^{m_{0}^{\prime}}
$$

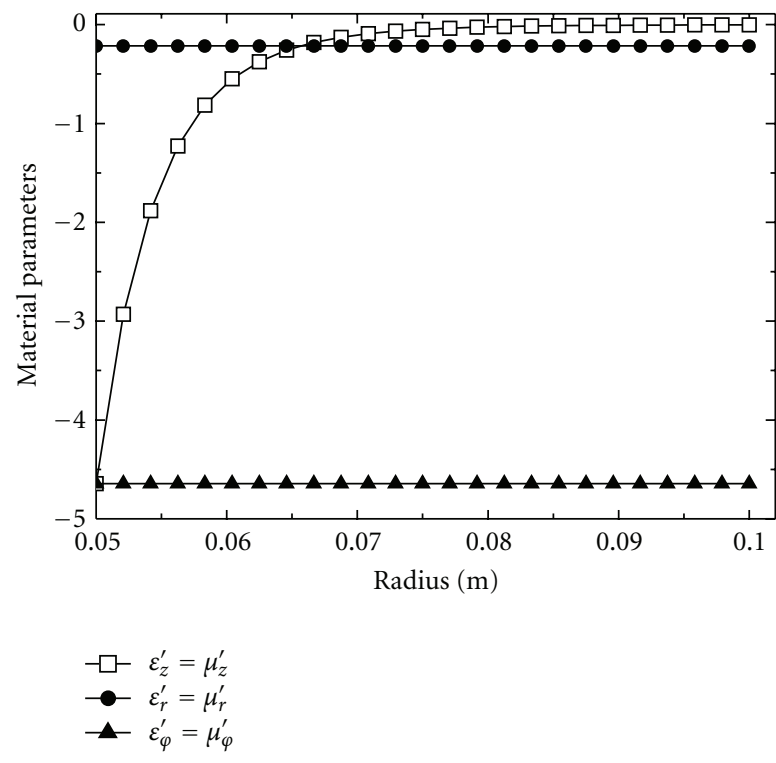

(a)

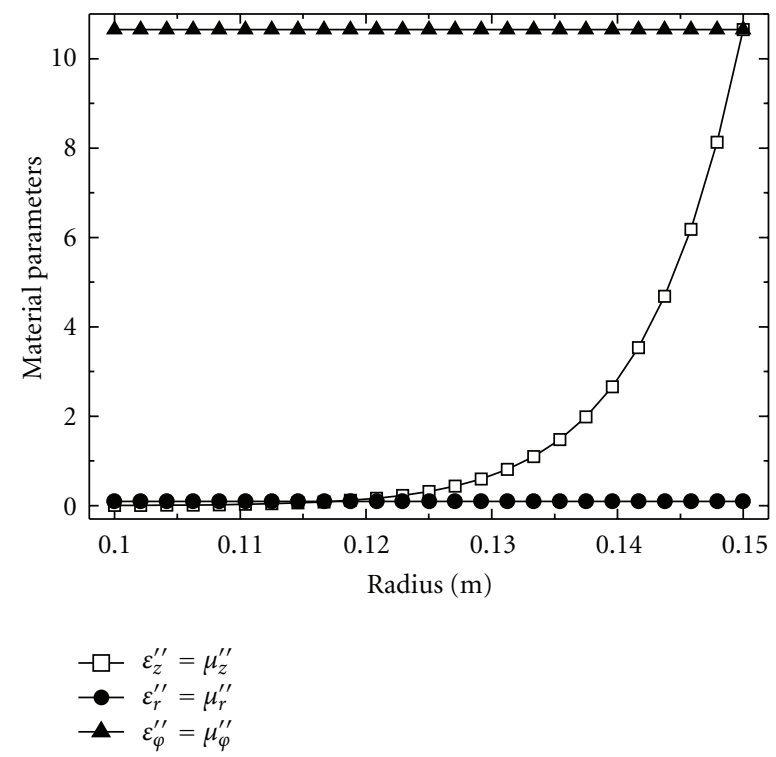

(b)

FIGURE 2: Constitutive parameters distribution along radial direction of the reciprocal cloak. (a) The complementary layer $\left(a<r^{\prime}<\right.$ $b)$. (b) The recovering layer $\left(b<r^{\prime \prime}<c\right)$.

where $m_{0}^{\prime}=\log _{(b / a)}(\delta / a), m_{1}^{\prime}=a^{\left(1-m_{0}^{\prime}\right)}$. Then, material parameters of the complementary media layer can be obtained as follows:

$$
\begin{gathered}
\frac{\varepsilon_{r}^{\prime}}{\varepsilon_{o r}}=\frac{\mu_{r}^{\prime}}{\mu_{o r}}=\frac{1}{m_{0}^{\prime}}, \\
\frac{\varepsilon_{\varphi}^{\prime}}{\varepsilon_{o \varphi}}=\frac{\mu_{\varphi}^{\prime}}{\mu_{o \varphi}}=m_{0}^{\prime}, \\
\frac{\varepsilon_{z}^{\prime}}{\varepsilon_{o z}}=\frac{\mu_{z}^{\prime}}{\mu_{o z}}=m_{0}^{\prime}\left(\frac{r^{\prime}}{a}\right)^{2\left(m_{0}^{\prime}-1\right)} .
\end{gathered}
$$




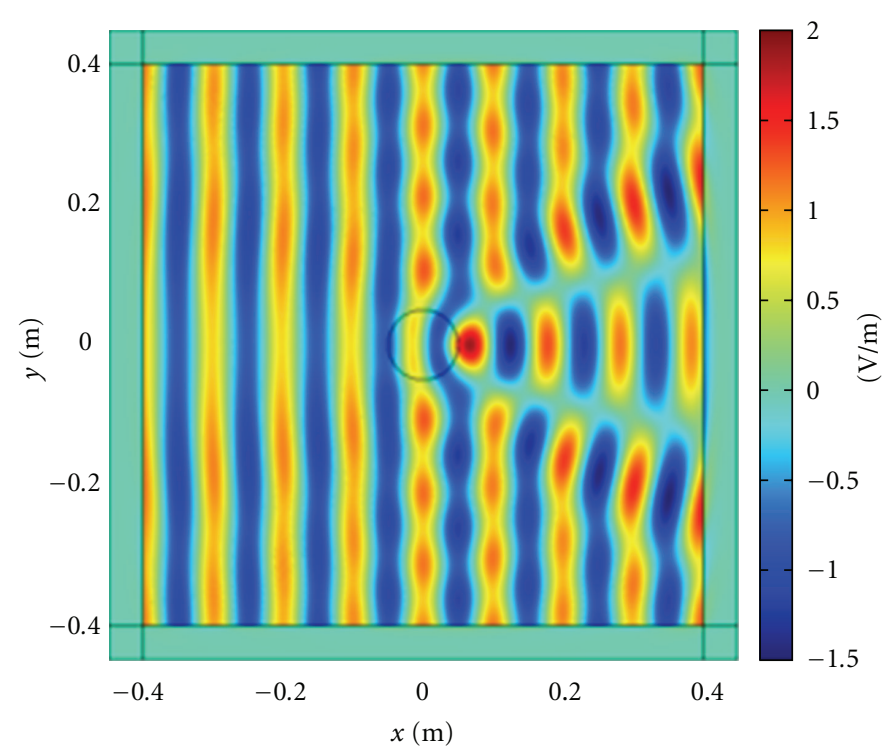

(a)

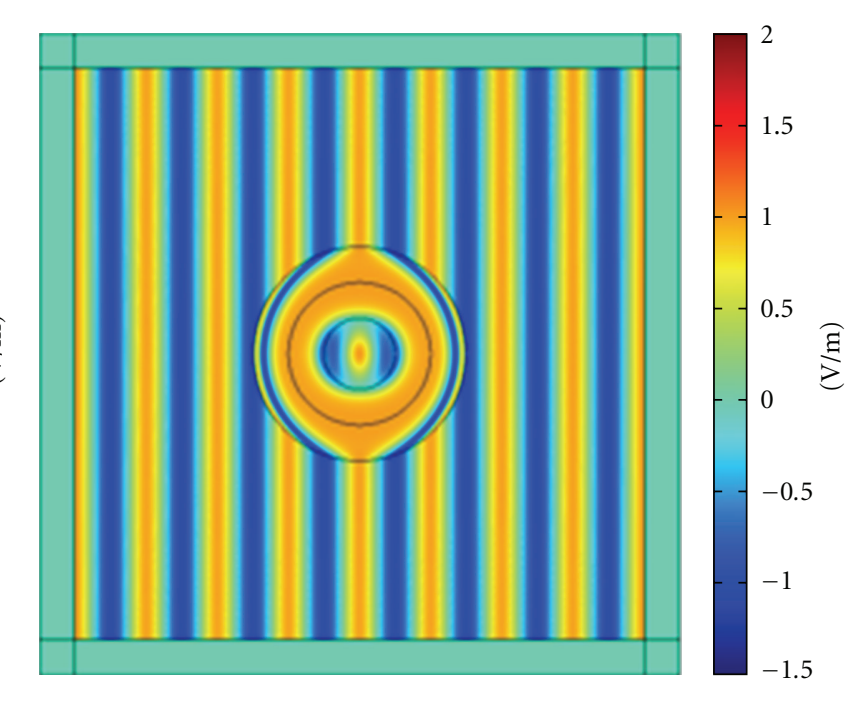

(b)

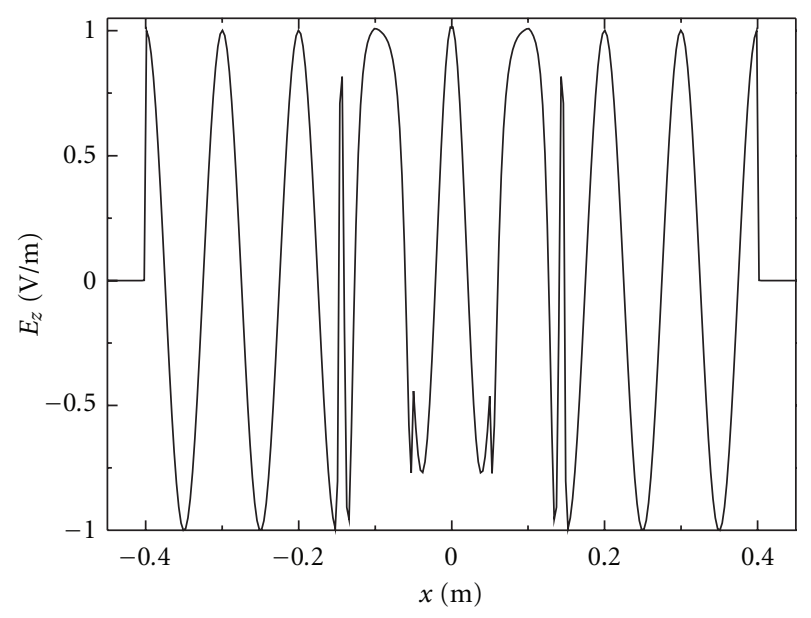

(c)

FIGURE 3: Electric field $\left(E_{z}\right)$ distributions in the computational domain. (a) Scattering pattern of a dielectric object with $\varepsilon_{z o}=2, \mu_{r o}=$ $1, \mu_{\varphi o}=1$. (b) The object is hidden by the reciprocal cloak. (c) Electric field distribution along $x$ axis.

In the recovering layer, we suppose the coordinate transformation equation is $r=f\left(r^{\prime \prime}\right)$, where $f\left(r^{\prime \prime}\right)$ satisfies $f(b)=\delta, f(c)=c$. Similarly, according to the preceding procedure, relative permittivity and permeability of the recovering layer can be obtained as

$$
\begin{gathered}
\varepsilon_{r}^{\prime \prime}=\mu_{r}^{\prime \prime}=\frac{1}{m_{0}^{\prime \prime},} \\
\varepsilon_{\varphi}^{\prime \prime}=\mu_{\varphi}^{\prime \prime}=m_{0}^{\prime \prime}, \\
\varepsilon_{z}^{\prime \prime}=\mu_{z}^{\prime \prime}=m_{0}^{\prime \prime}\left(\frac{r^{\prime \prime}}{c}\right)^{2\left(m_{0}^{\prime \prime}-1\right)},
\end{gathered}
$$

where $m_{0}^{\prime \prime}=\log _{(b / c)}(\delta / c)$. With the geometry parameters of $a=0.05 \mathrm{~m}, b=0.1 \mathrm{~m}, c=0.15 \mathrm{~m}$, constitutive parameters of the reciprocal cloak can be calculated through (4) and (5), as shown in Figure 2. It is seen that the complementary layer is a double negative shell, while all components in the recovering layer are positive. Moreover, both $r$ and $\varphi$ components in the complementary layer and the recovering layer are constants; only $z$ component is dependent on the radius of the reciprocal cloak.

\section{Results and Discussions}

In this section, performance of the reciprocal cloak is simulated based on the commercia software COMSOL Multiphysics. We consider the case of transverse-electric (TE) polarization, and only $\varepsilon_{z}, \mu_{r}, \mu_{\varphi}$ components of the material parameters are required. The incident wave with electric field polarized along $z$ axis is impinging onto the cloak. Frequency of the incident wave is set to be $3 \mathrm{GHz}$. In the simulation, the whole computational domain is surrounded by a perfectly matched layer that absorbs the outward traveling waves. 


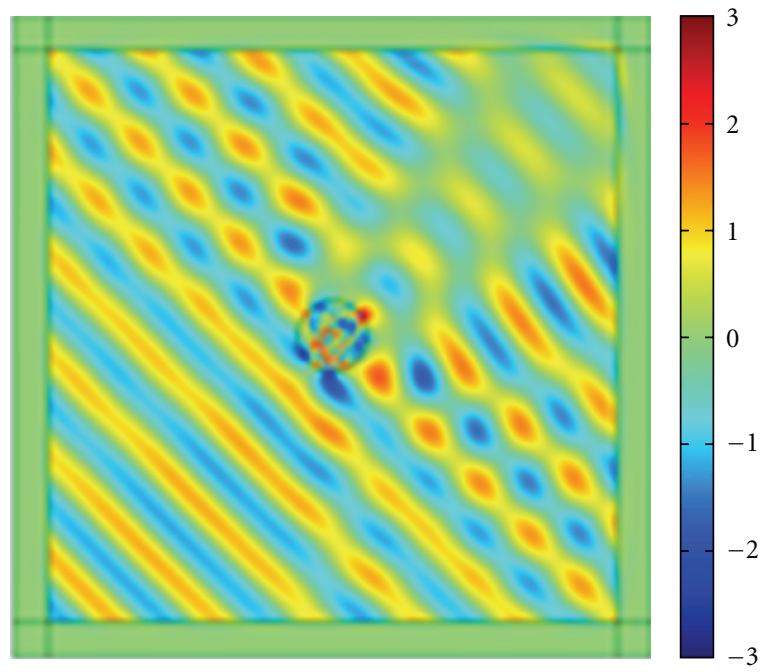

(a)

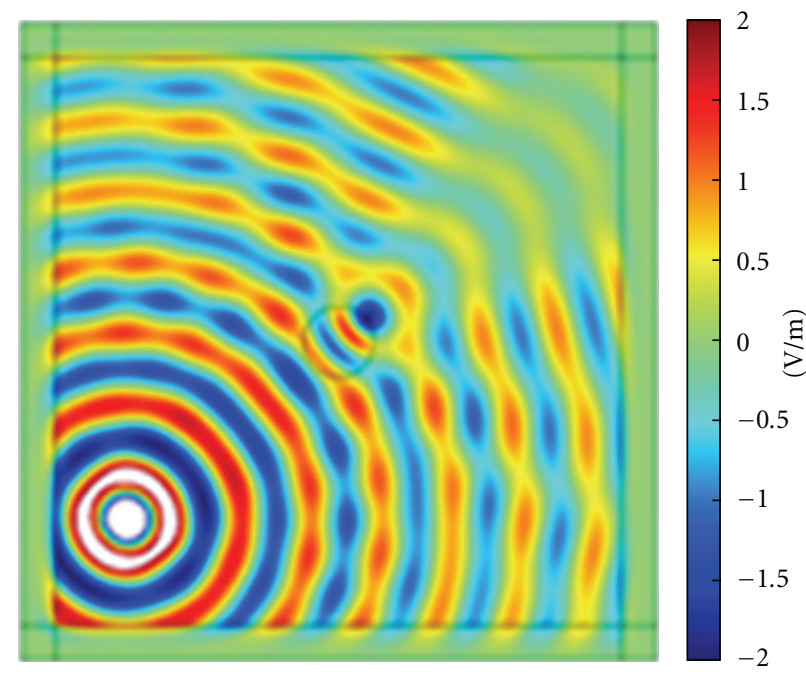

(c)

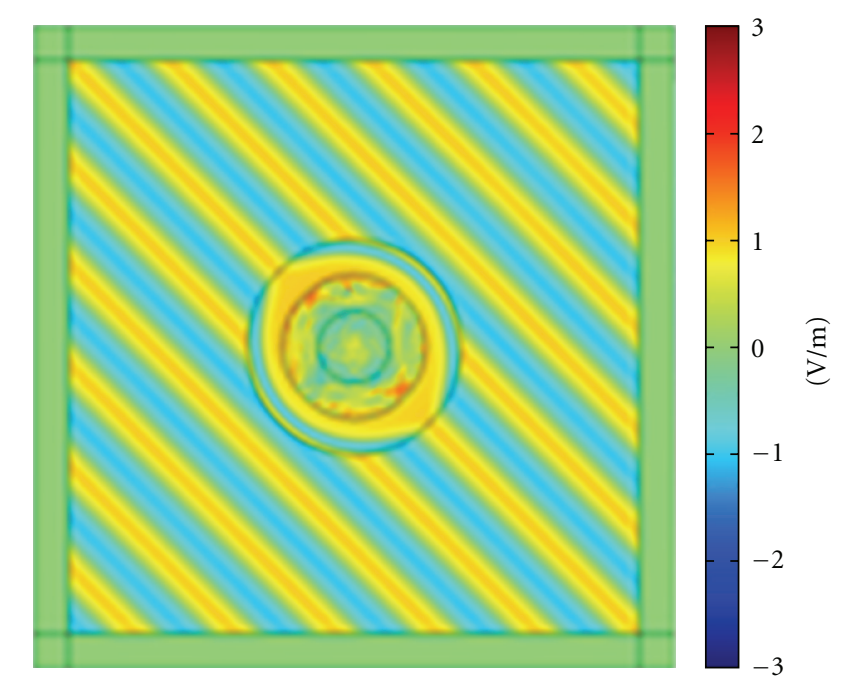

(b)

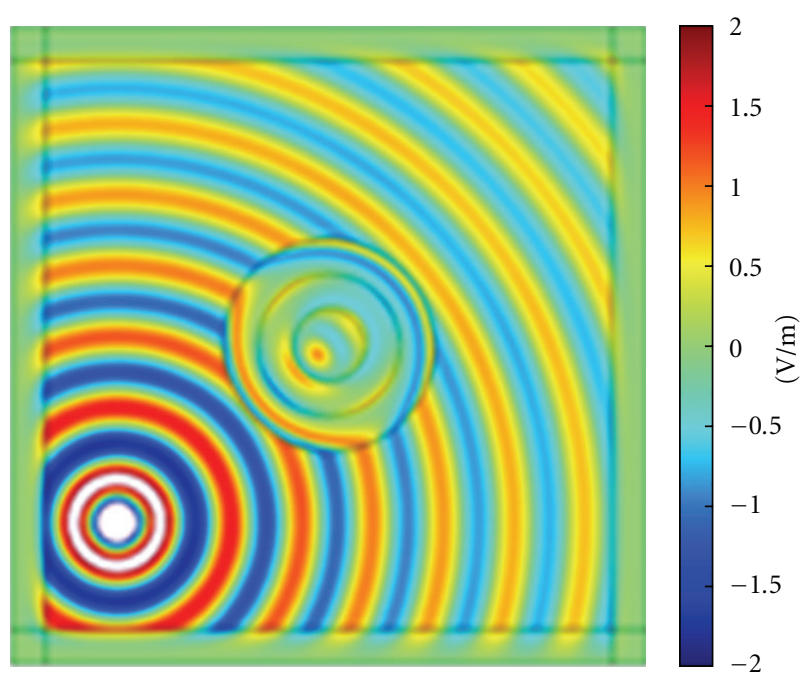

(d)

FIgURE 4: Electric field $\left(E_{z}\right)$ distributions in the computational domain. (a) Scattering pattern of the object with anisotropic permeability $\left(\mu_{r o}=-1, \mu_{\varphi o}=2\right)$. The plane wave is incident at an angle of $45^{\circ}$. (b) The object in (a) is hidden by the reciprocal cloak. (c) Scattering pattern of an object with linearly changing permittivity $\left(\varepsilon_{z o}=2 r+3\right)$. The current source with amplitude of $10^{-3} \mathrm{~A}$ is located at $(-0.3 \mathrm{~m}$, $-0.25 \mathrm{~m}$ ). (d) The object in (c) is hidden by the reciprocal cloak.

Figure 3(a) shows the scattering pattern of a dielectric circular cylinder with material parameters of $\varepsilon_{z o}=2, \mu_{r o}=$ $1, \mu_{\varphi o}=1$. In order to make it invisible, we put it inside a reciprocal cloak, as shown in Figure 3(b). The incident plane wave with unit amplitude propagates from left to right along $x$ axis. The perfect recovered wavefront demonstrates the effectiveness of the cloak. Figure 3(c) plots the electric field distribution along $x$ axis of the computational domain. We can clearly observe that the electric field intensity inside the cloak is equal to that outside. It indicates that the object enclosed inside the reciprocal cloak can receive information from the outside without distortion, while its presence cannot be detected. It is worth noting that arbitrary object with isotropic or anisotropic material parameters can be cloaked as long as the complementary media layer is "custom-made" according to (4), and the performance of the cloak is independent on the direction of the incident wave and the excitation source. For example, in Figures 4(a) and $4(\mathrm{~b})$, we demonstrate the cloaking of the object with anisotropic permeability $\mu_{r o}=-1, \mu_{\varphi o}=2$. The plane wave is incident at an angel of $45^{\circ}$. Besides, the object with linear changing permittivity, such as $\mathcal{E}_{z o}=2 r+3$, can also be cloaked under cylindrical wave irradiation, as shown in Figures 4(c) and 4(d).

Since electromagnetic wave penetrates into the cloaking region, the hidden object such as an antenna or a sensor can receive information from the outside. This indicates that the reciprocal cloak may have potential application for 


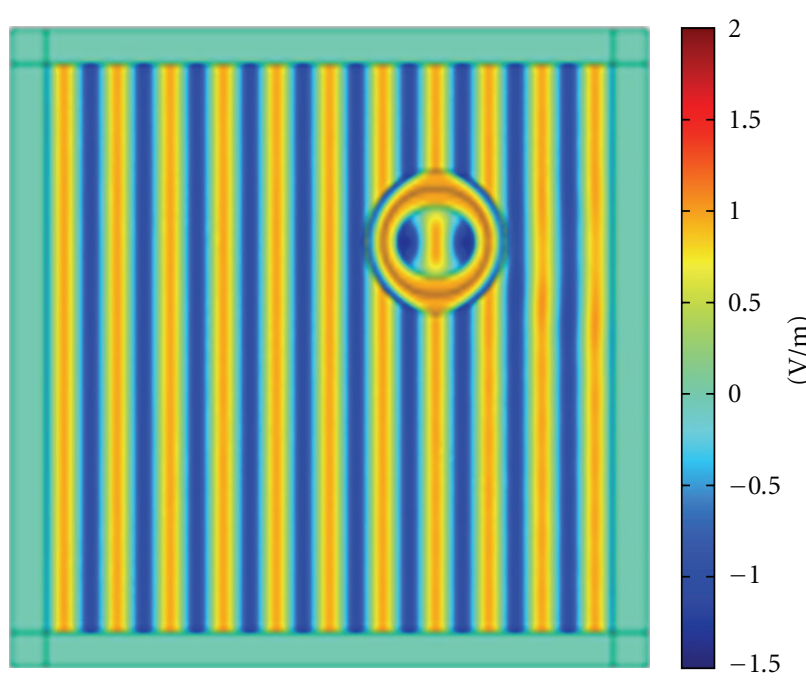

(a)

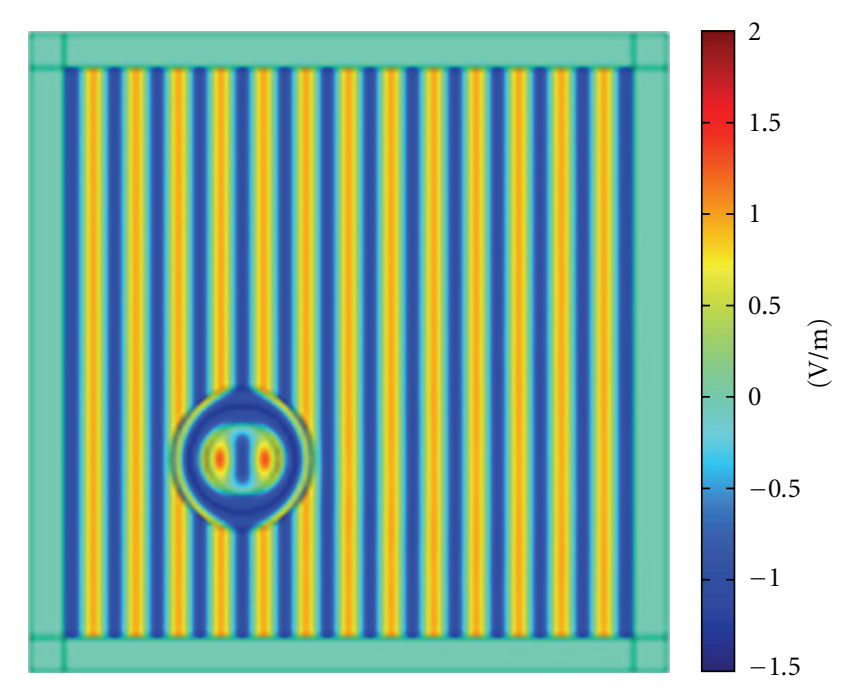

(b)

Figure 5: (a) Cloak A with working frequency of $4 \mathrm{GHz}$. (b) Cloak B with working frequency of $5 \mathrm{GHz}$.

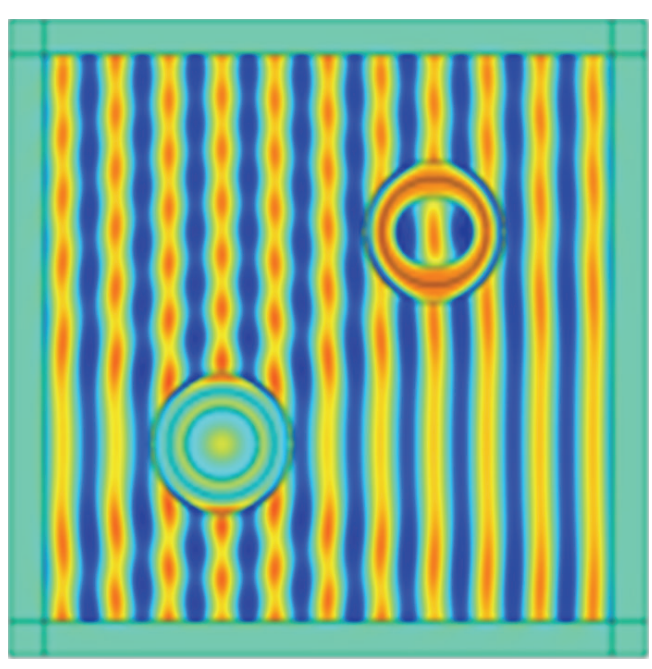

(a)

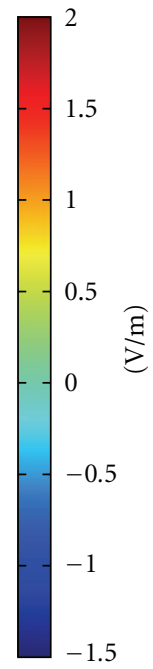

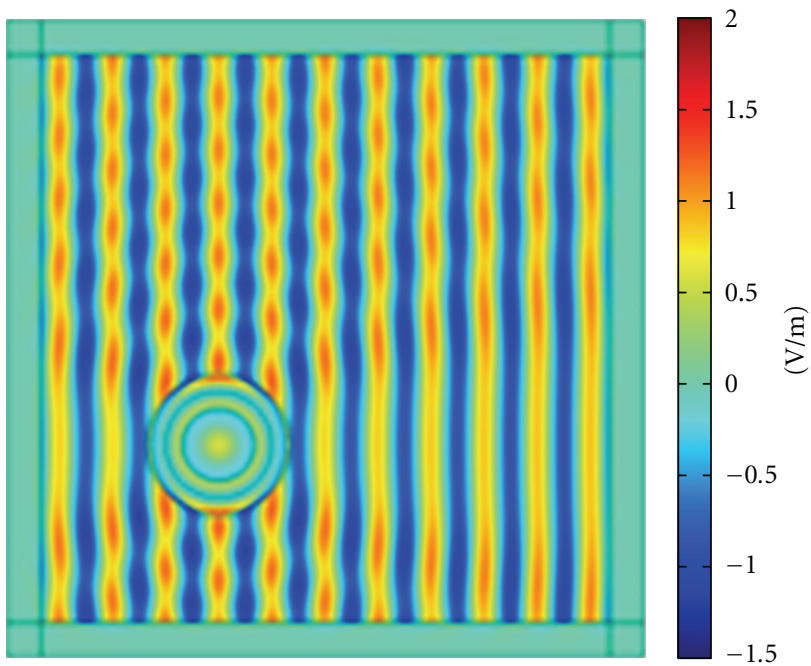

(b)

Figure 6: (a) Electric field distribution in the computational domain of the cloaking system. (b) Cloak B at disabled state.

enhancing the electromagnetic compatibility of an electronic device. To demonstrate the EMC application of the cloak, we simulate a cloaking system, which is consisted of two close-ly neighboring reciprocal cloaks. In order to assess the frequency behavior of the cloaking system, the Drude dispersion model has been employed for the double-negative shell of the cloak. In (6), the quantity $\omega_{p}$ is the plasma frequency, and it is chosen such that negative permittivity and permeability are obtained at the respective design frequency. The parameter $\Gamma$ is the collision frequency representing the losses in the material:

$$
\varepsilon(\mu)=1-\frac{\omega_{p}^{2}}{\omega(\omega+i \Gamma)} .
$$

Figures 5(a) and 5(b) show the simulation results of two reciprocal cloaks with working frequency of $4 \mathrm{GHz}$ (Cloak A) and $5 \mathrm{GHz}$ (Cloak B), respectively. Now we put the two cloaks together and set the frequency of the impinging plane wave to be $4 \mathrm{GHz}$ which is the working frequency of cloak A. Electric field distribution of the cloaking system is shown in Figure 6(a). As a comparison, electric field distribution in the computational domain of Cloak B at the disabled state is also simulated and shown in Figure 6(b). It is seen that field patterns in the two figures are the same. That means when Cloak A is at the working state, it will be invisible and it does not influence the field pattern of Cloak B. Contrarily, when Cloak B is at the working state, it will be invisible and it does not influence field pattern of Cloak A. Therefore, the two 


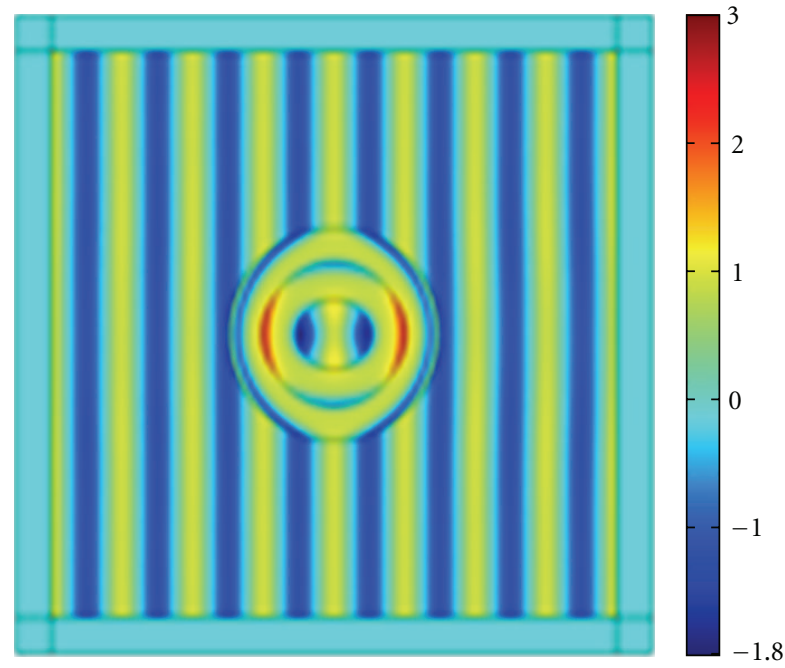

(a)

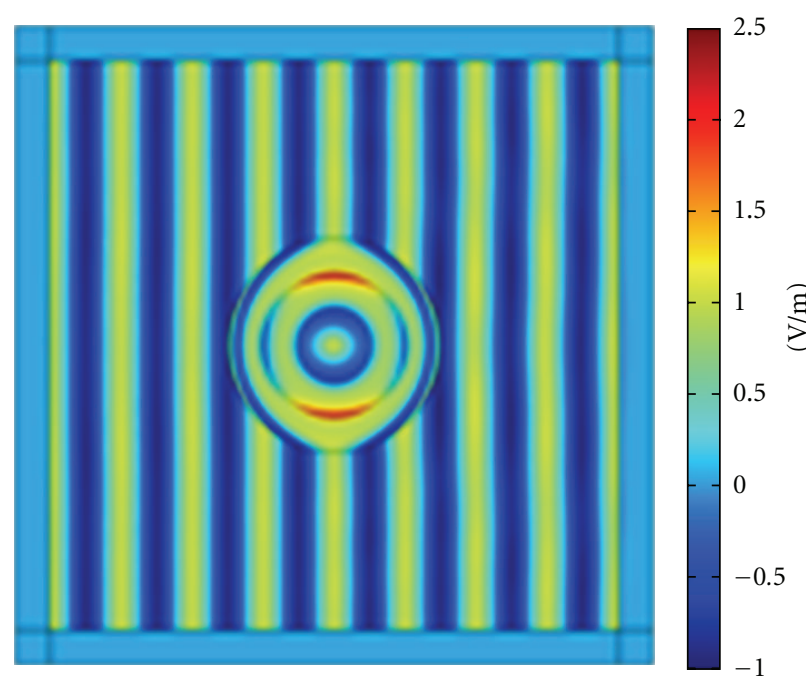

(c)

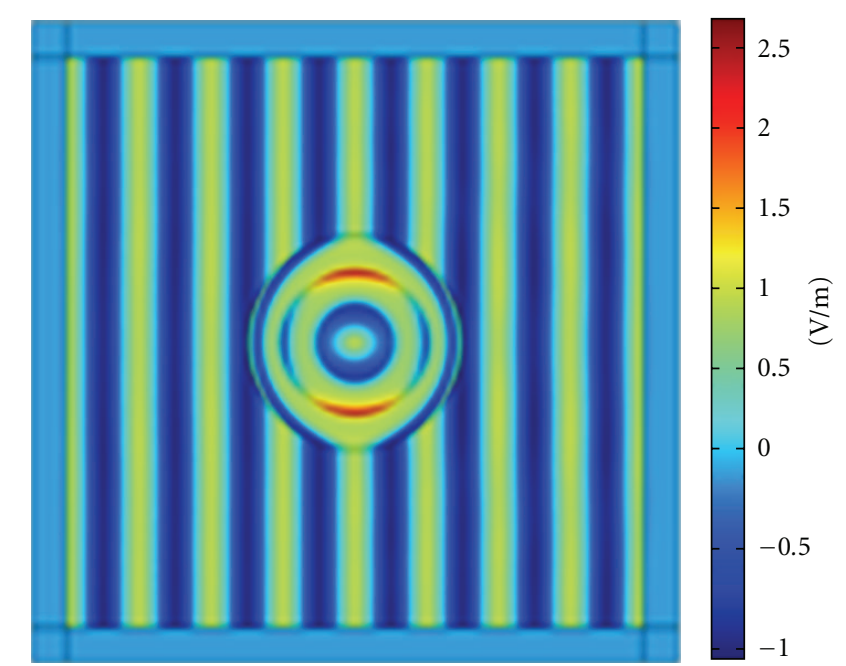

(b)

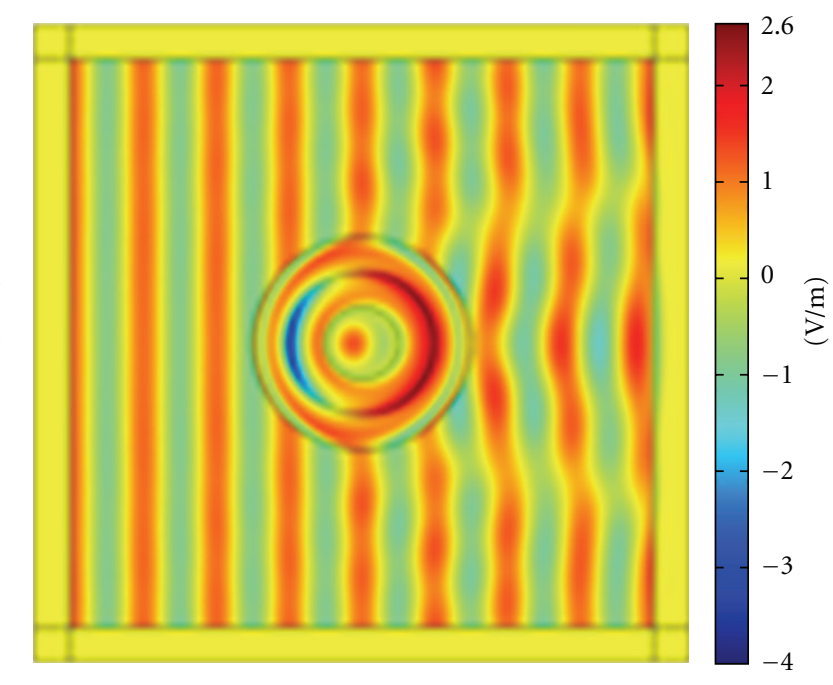

(d)

Figure 7: The electric field $\left(E_{z}\right)$ distributions in the vicinity of the cloak with loss tangents of 0.001 (a), 0.01 (b), 0.015 (c), and 0.1 (d), respectively.

closely neighboring reciprocal cloaks do not interfere with each other. If the reciprocal cloaks were applied to protect an antenna array, mutual interference could be avoided.

In practical applications, metamaterials are always lossy. Thus, in what follows, we investigate the influence of loss tangent on the performance of the cloak, and the material parameter deviation is also considered. Electric field distribution in the vicinity of the reciprocal cloak with electric and magnetic loss tangents of $0.001,0.01,0.015$, and 0.1 are displayed in Figures $7(\mathrm{a}), 7(\mathrm{~b}), 7(\mathrm{c})$, and $7(\mathrm{~d})$. Here, material parameters of the cloaked object are set to be $\varepsilon_{z o}=$ $2, \mu_{r o}=1, \mu_{\varphi o}=1$. From Figures 7(a)-7(c), we can observe that field distribution is basically undisturbed when loss tangent is less then 0.015; but when the loss tangents of metamaterials is 0.1 or more than that, it deteriorates the performance of the reciprocal cloak especially in the forward scattering region, as shown in Figure 7(d). Therefore, loss tangents of metamaterials being less than or equal to 0.015 is acceptable.

To investigate the influence of material parameter deviation on cloaking effect, we multiply transverse constitutive material parameters $\left(\mu_{r}, \mu_{\varphi}\right)$ for both the complementary media layer and recovering layer by coefficient $\delta_{1}$, and axial constitutive material parameter $\left(\varepsilon_{z}\right)$ by coefficient $\delta_{2}$. This represents the slight changes in the transverse and axial material parameters, respectively. When there is a slight change in the axial parameter, the following three cases are considered: (i) the transverse parameters are kept invariant, (ii) the impedance $\left(Z=\sqrt{\mu_{\varphi} / \varepsilon_{z}}\right.$ and $\left.\sqrt{\mu_{r} / \varepsilon_{z}}\right)$ is kept invariant, (iii) the refractive index $\left(n=\sqrt{\mu_{\varphi} \varepsilon_{z}}\right.$ and $\left.\sqrt{\mu_{r} \varepsilon_{z}}\right)$ is kept invariant. The corresponding changes in the transverse parameters can 

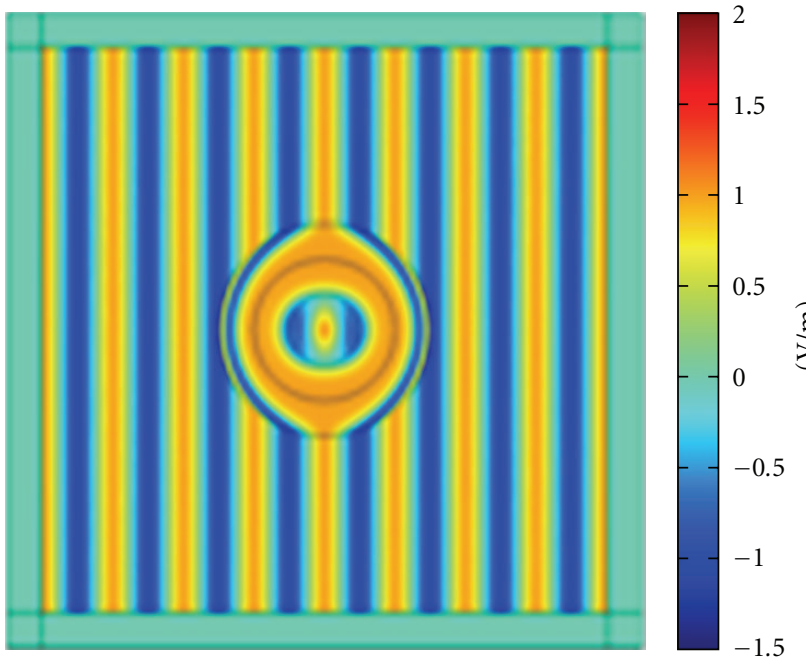

(a)

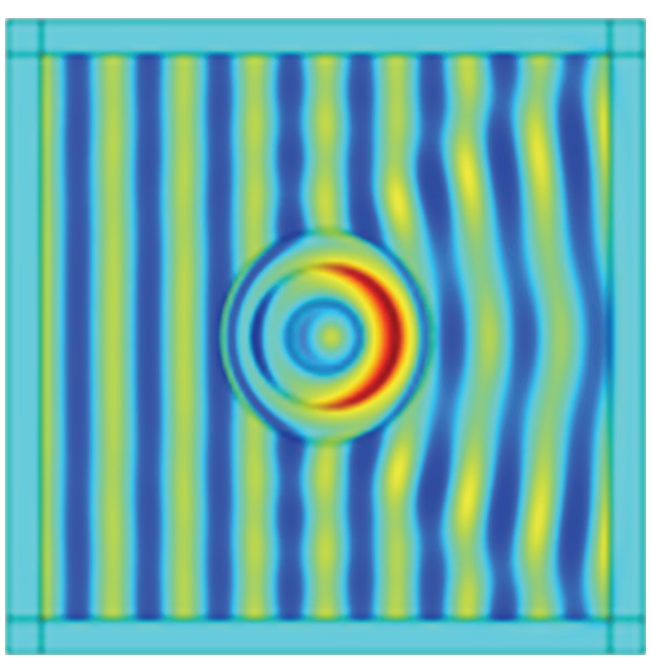

(c)

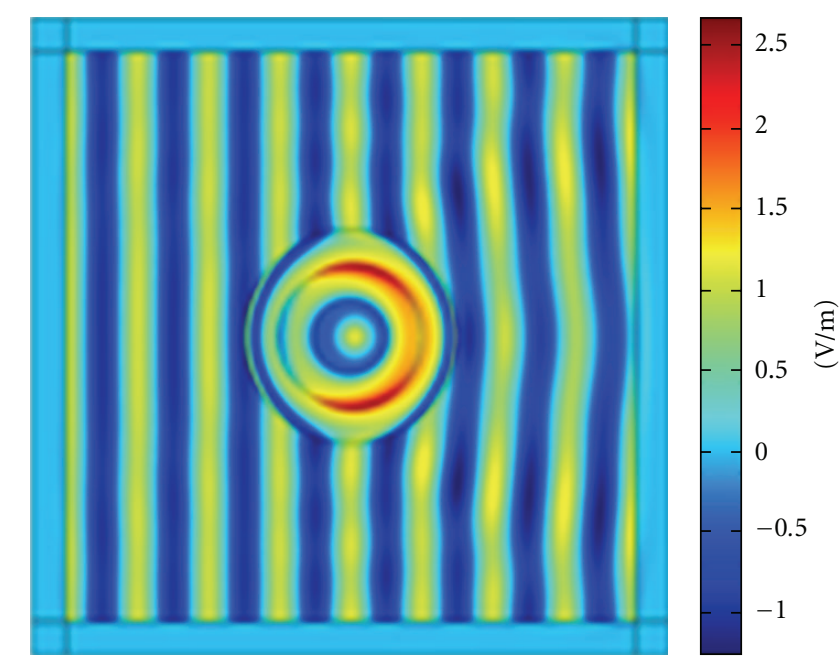

(b)
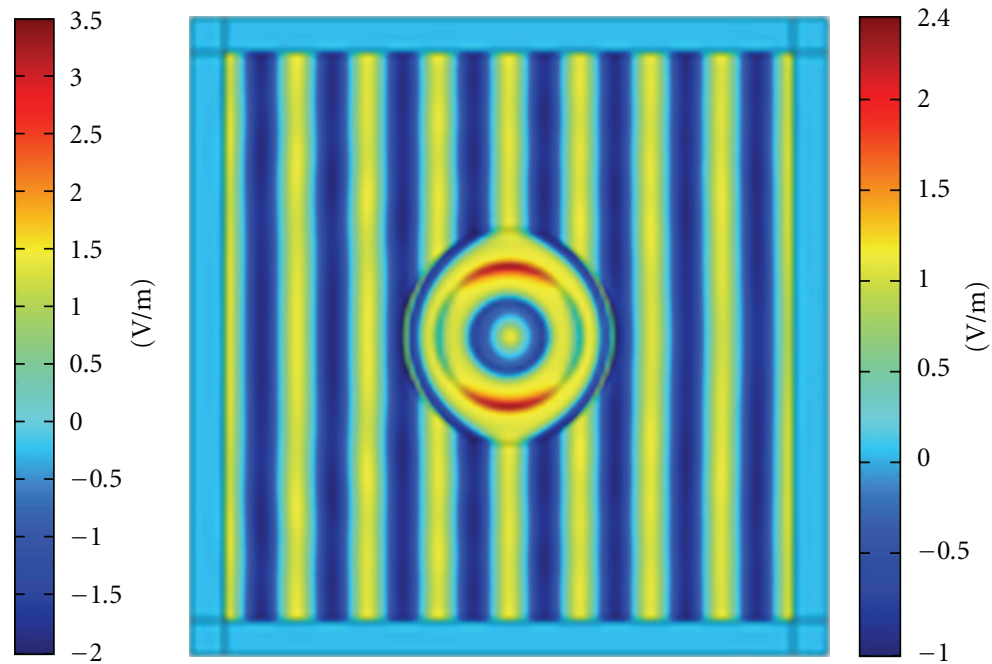

(d)

FIGURE 8: Electric field $\left(E_{z}\right)$ distributions in the vicinity of the reciprocal cloak without perturbation $(\mathrm{a})$, with negative perturbation $\left(\delta_{2}=\right.$ $0.9)$ while transverse parameters are invariant $\left(\delta_{1}=1\right)(\mathrm{b})$, impedances are invariant $\left(\delta_{1}=0.9\right)(\mathrm{c})$, and refraction index is invariant $\left(\delta_{1}=1 / 0.9\right)(\mathrm{d})$.

be easily derived. In the simulation, the negative perturbation $\left(\delta_{2}=0.9\right)$ and positive perturbation $\left(\delta_{2}=1.1\right)$ in the axial parameter are taken into consideration, and the material parameters of circular dielectric object to be cloaked is $\varepsilon_{z o}=2, \mu_{r o}=1, \mu_{\varphi o}=1$. Figures 8(b)-8(d) and Figures 9(b) -9 (d) show the corresponding electric field distributions in the vicinity of the reciprocal cloak. Simulation results for the cloak without perturbation are also simulated for comparisons, as shown in Figures 8(a) and 9(a). It is clear that whether the transverse parameters change or not, the performance of the reciprocal cloak will be affected by the positive or negative perturbation in the axial parameters (as shown in Figures 8(b)-8(c), and Figures 9(b)-9(c)). In practice, if we want to keep the cloaking effects of the reciprocal cloak while at the same time minimizing its scattering field, it is the best choice to keep the refractive index invariant (shown in Figures 8(d) and 9(d)).

\section{Conclusions}

In conclusion, a cylindrical electromagnetic reciprocal cloak with only axial material parameter spatially variant is proposed and designed by means of transformation optics. The EMC ability of the cloak is demonstrated based on full wave simulation. Besides, it is found that the reciprocal cloak is robust against the perturbation in the axial material parameters when the refractive index is kept invariant. The simplicity of the material parameters would put a step further towards the practical realization the reciprocal cloak, which shows some advantages in opening up an avenue for 


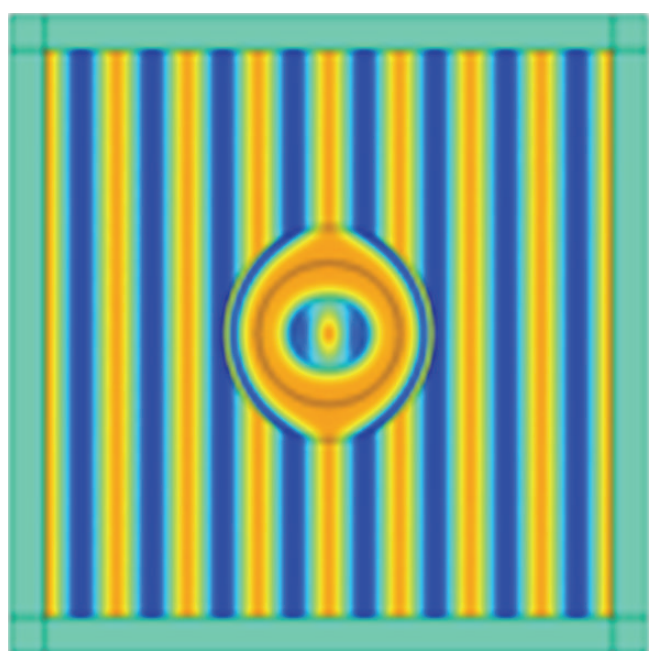

(a)

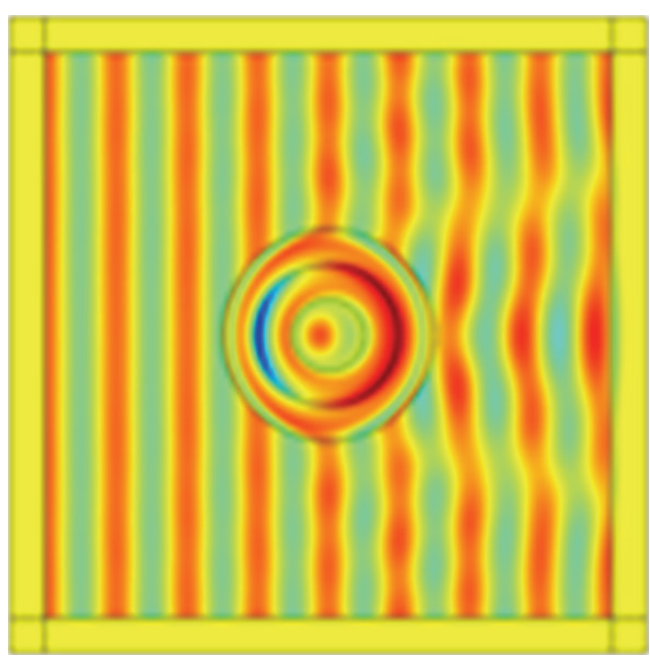

(c)

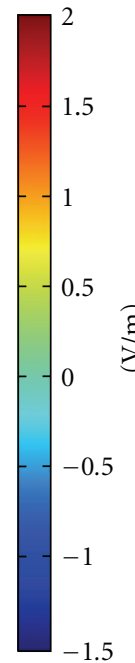

2.6

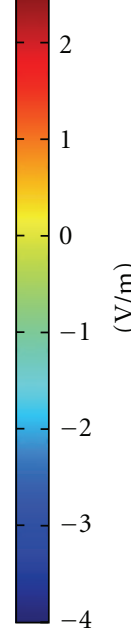

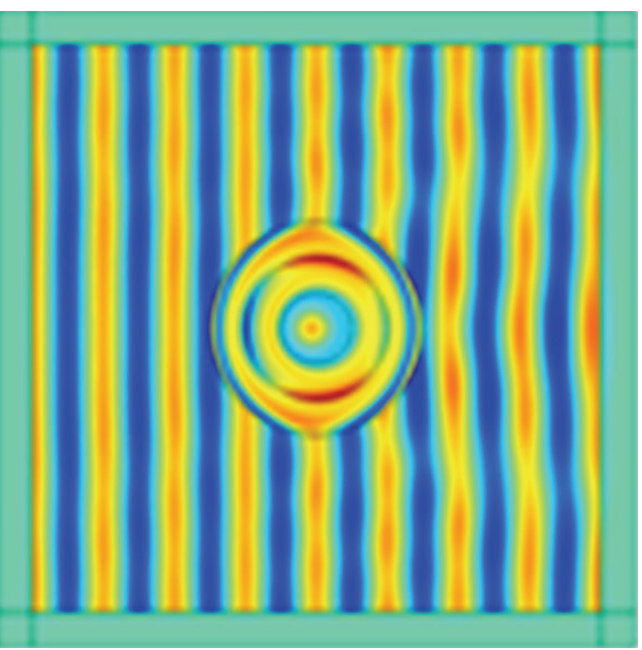

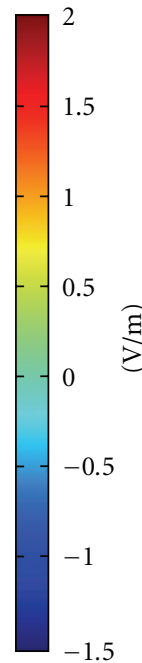

(b)

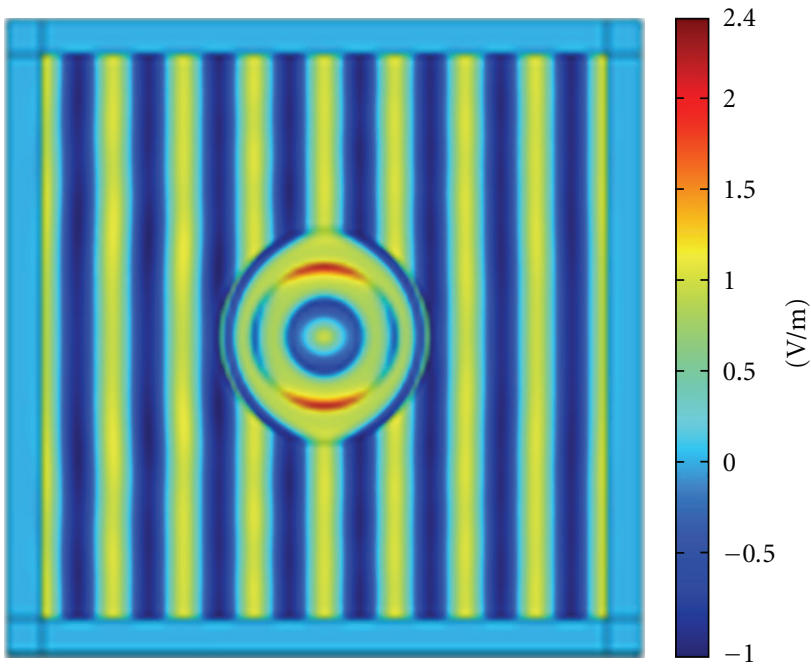

(d)

Figure 9: Electric field $\left(E_{Z}\right)$ distributions in the vicinity of the reciprocal cloak without perturbation (a), with positive perturbation $\left(\delta_{2}=\right.$ 1.1) while transverse parameters are invariant $\left(\delta_{1}=1\right)(\mathrm{b})$, impedances are invariant $\left(\delta_{1}=1.1\right)$ (c), and refraction index is invariant $\left(\delta_{1}=1 / 1.1\right)(\mathrm{d})$.

designing antennae and sensors with perfect electromagnetic compatibility.

\section{Acknowledgments}

This work was supported by the National Natural Science Foundation of China (Grant no. 60861002, 61161007, 61162004), the Natural Science Foundation of Yunnan Province (Grant no. 2011FB018), and NSFC-YN (Grant no. U1037603).

\section{References}

[1] V. G. Veselago, "The electrodynamics of substances with simultaneously negative values of $\varepsilon$ and $\mu$," Soviet Physics Uspekhi, vol. 10, no. 4, pp. 509-514, 1968.
[2] J. B. Pendry, "Negative refraction makes a perfect lens," Physical Review Letters, vol. 85, no. 18, pp. 3966-3969, 2000.

[3] J. J. Yang, M. Huang, and J. Sun, "Double negative metamaterial sensor based on microring resonator," IEEE Sensors Journal, vol. 11, no. 10, pp. 2254-2259, 2011.

[4] M. Huang, J. Yang, S. Jun, S. Mu, and Y. Lan, "Simulation and analysis of a metamaterial sensor based on a microring resonator," Sensors, vol. 11, no. 6, pp. 5886-5899, 2011.

[5] M. Huang, J. Yang, J. Sun, J. Shi, and J. Peng, "Modelling and analysis of $\omega$-shaped double negative material-assisted microwave sensor," Journal of Infrared, Millimeter, and Terahertz Waves, vol. 30, no. 11, pp. 1131-1138, 2009.

[6] A. Azarbar and J. Ghalibafan, "A compact low-permittivity dual-layer EBG structure for mutual coupling reduction," International Journal of Antennas and Propagation, vol. 2011, Article ID 237454, 6 pages, 2011. 
[7] J. B. Pendry, D. Schurig, and D. R. Smith, "Controlling electromagnetic fields," Science, vol. 312, no. 5781, pp. 1780-1782, 2006.

[8] U. Leonhardt, "Optical conformal mapping," Science, vol. 312, no. 5781, pp. 1777-1780, 2006.

[9] D. Schurig, J. J. Mock, B. J. Justice et al., "Metamaterial electromagnetic cloak at microwave frequencies," Science, vol. 314, no. 5801, pp. 977-980, 2006.

[10] X. Liu, C. Li, K. Yao et al., "Experimental verification of broadband invisibility using a cloak based on inductor-capacitor networks," Applied Physics Letters, vol. 95, no. 19, Article ID 191107, 2009.

[11] P. Alitalo and S. Tretyakov, "Electromagnetic cloaking with metamaterials," Materials Today, vol. 12, no. 3, pp. 22-29, 2009.

[12] U. Leonhardt and T. Philbin, Geometry and Light: The Science of Invisibility, Dover Publications, Mineola, NY, USA, 2010.

[13] U. Leonhardt and T. G. Philbin, "General relativity in electrical engineering," New Journal of Physics, vol. 8, article no. 247, 2006.

[14] J. Yang, M. Huang, C. Yang, Z. Xiao, and J. Peng, "Metamaterial electromagnetic concentrators with arbitrary geometries," Optics Express, vol. 17, no. 22, pp. 19656-19661, 2009.

[15] J. J. Yang, T. H. Li, M. Huang, and M. Cheng, "Transparent device with homogeneous material parameters," Applied Physics A, vol. 104, no. 2, pp. 733-737, 2011.

[16] W. X. Jiang, J. Y. Chin, and T. J. Cui, “Anisotropic metamaterial devices," Materials Today, vol. 12, no. 12, pp. 26-33, 2009.

[17] H. Chen, C. T. Chan, and P. Sheng, "Transformation optics and metamaterials," Nature Materials, vol. 9, no. 5, pp. 387396, 2010.

[18] A. E. Dubinov and L. A. Mytareva, "Invisible cloaking of material bodies using the wave flow method," Physics-Uspekhi, vol. 53, no. 5, pp. 455-479, 2010.

[19] H. Ma, S. Qu, Z. Xu, and J. Wang, “The open cloak," Applied Physics Letters, vol. 94, no. 10, Article ID 103501, 2009.

[20] T. C. Han, C. W. Qiu, and X. H. Tang, "Creating rigorous open cloaks," Journal of Electromagnetic Waves and Applications, vol. 24, no. 13, pp. 1839-1847, 2010.

[21] T. Han, C. Qiu, and X. Tang, "The general two-dimensional open-closed cloak with tunable inherent discontinuity and directional communication," Applied Physics Letters, vol. 97, no. 12, Article ID 124104, 2010.

[22] Y. Lai, H. Chen, Z. Q. Zhang, and C. T. Chan, "Complementary media invisibility cloak that cloaks objects at a distance outside the cloaking shell," Physical Review Letters, vol. 102, no. 9, Article ID 093901, 2009.

[23] C. Yang, J. Yang, M. Huang, Z. Xiao, and J. Peng, "An external cloak with arbitrary cross section based on complementary medium and coordinate transformation," Optics Express, vol. 19, no. 2, pp. 1147-1157, 2011.

[24] H. Chew and M. Kerker, "Abnormally low electromagnetic scattering cross sections," Journal of the Optical Society of America, vol. 66, no. 5, pp. 445-449, 1976.

[25] A. Alù and N. Engheta, "Achieving transparency with plasmonic and metamaterial coatings," Physical Review E, vol. 72, no. 1, Article ID 016623, 2005.

[26] L. Gao, T. H. Fung, K. W. Yu, and C. W. Qiu, "Electromagnetic transparency by coated spheres with radial anisotropy," Physical Review E, vol. 78, no. 4, Article ID 046609, 2008.

[27] Y. Ni, L. Gao, and C. W. Qiu, "Achieving invisibility of homogeneous cylindrically anisotropic cylinders," Plasmonics, vol. 5, no. 3, pp. 251-258, 2010.
[28] J. J. Yang, M. Huang, C. F. Yang, and J. Yu, "Reciprocal invisibility cloak based on complementary media," European Physical Journal D, vol. 61, no. 3, pp. 731-736, 2011.

[29] B. Wood, J. B. Pendry, and D. P. Tsai, "Directed subwavelength imaging using a layered metal-dielectric system," Physical Review B, vol. 74, no. 11, Article ID 115116, 2006.

[30] Y. Huang, Y. Feng, and T. Jiang, "Electromagnetic cloaking by layered structure of homogeneous isotropic materials," Optics Express, vol. 15, no. 18, pp. 11133-11141, 2007. 

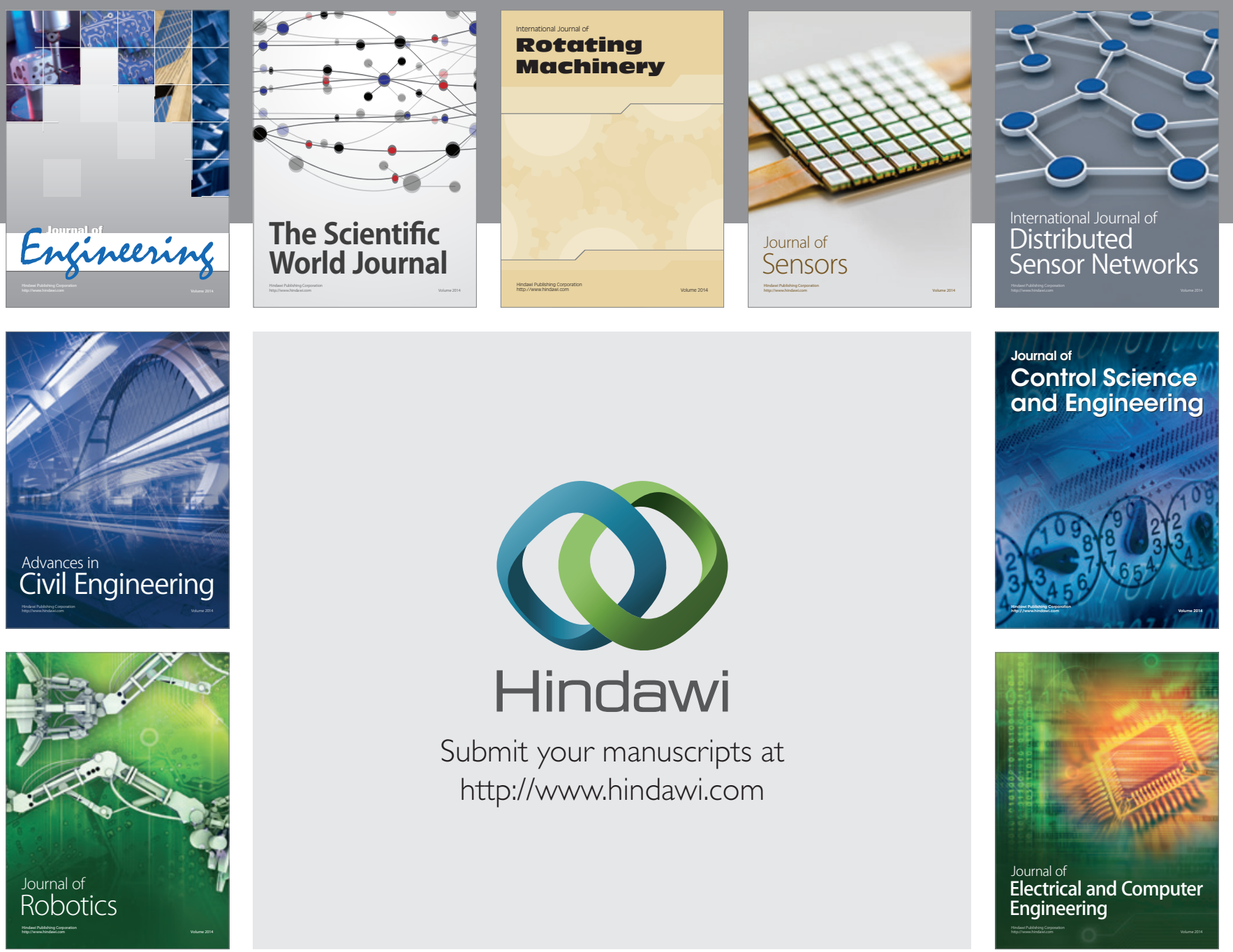

Submit your manuscripts at

http://www.hindawi.com
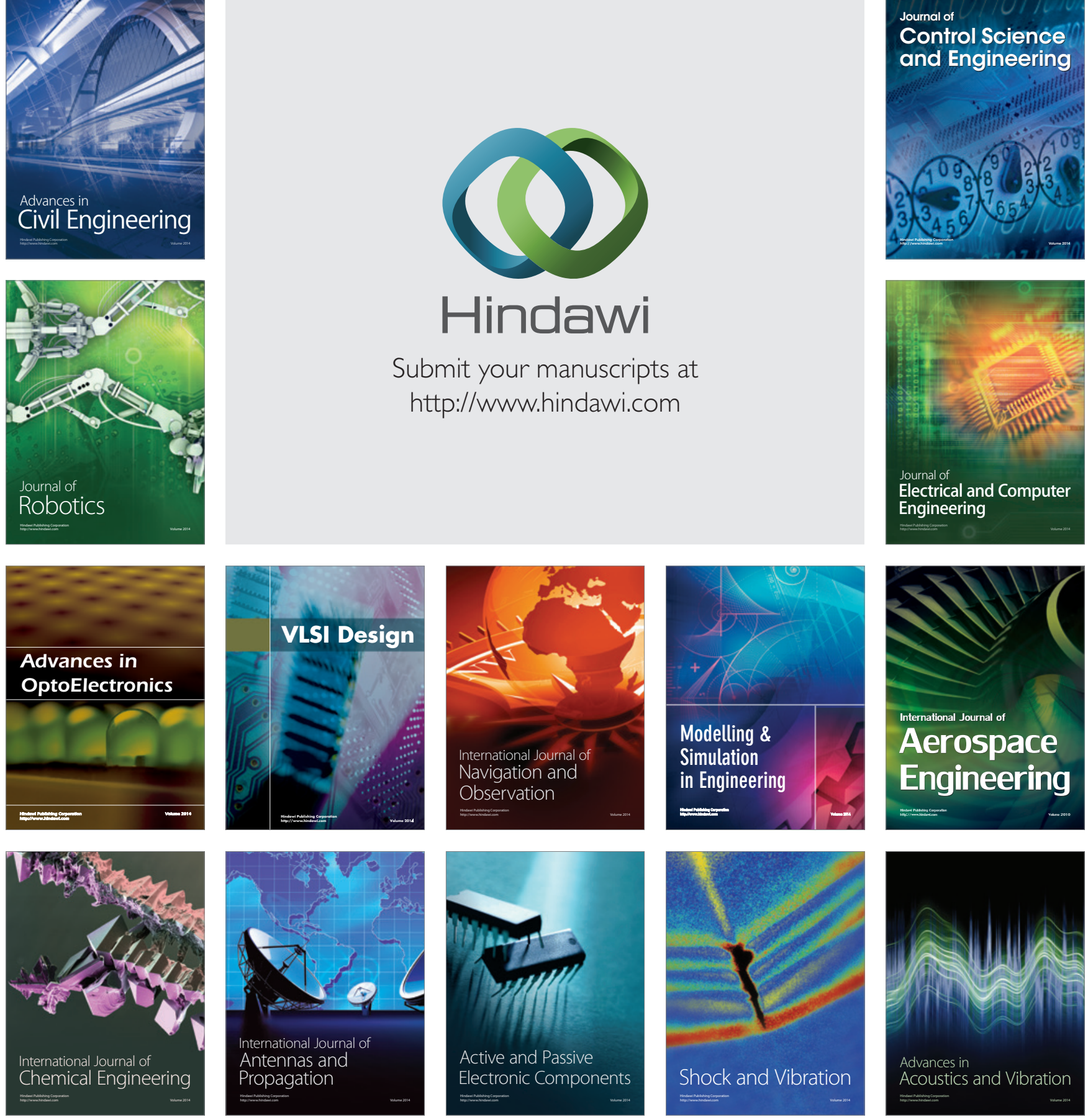